\title{
Comparative Expression Pattern of Two Vestigial-Like 2 Genes in Zebrafish
}

\author{
Aline Hamade ${ }^{1, *}$, Francine Rizk ${ }^{1}$, Carine Genet ${ }^{2}$, Xavier Cousin ${ }^{3,4}$ \\ ${ }^{1}$ Laboratoirede Physiopathologies Cellulaires et Moléculaires, EDST, Université Libanaise, Lebanon \\ ${ }^{2}$ INRA, UMR 1313 Génétique Animale et Biologie Intégrative, Jouy-en-Josas, France \\ ${ }^{3}$ Ifremer, Laboratoire d'Ecotoxicologie, Place Gaby Coll, BP 7, 17137 L'Houmeau, France \\ ${ }^{4}$ INRA, LPGP, Campus Beaulieu, 35042 Rennes Cedex, France \\ *Corresponding author: aline.hamade@ul.edu.lb
}

Copyright (C) 2013 Horizon Research Publishing All rights reserved.

\begin{abstract}
The vestigial $\mathrm{Vg}$ gene, initially characterized in Drosophila, encodes a transcription co-factor which is crucial for wings development. Vg binds via its Sd interaction domain (SID) to the Scalloped (Sd) transcription factor and its vertebrate homolog Tef1. Previous studies identified several vertebrate genes sharing high homology with Drosophila Vg SID such as Vgl (for Vg-like), TONDU (also known as Vgl1) or Vito-1 (also known as Vgl2). In order to investigate the role of vestigial-like 2 ( $\mathrm{vgll}$ ) in zebrafish muscle development, we managed to clone and characterize two zebrafishVgll2 homolog genes, Vgll2a and $V g l l 2 b$. Alignment data showed high sequence homology of Vgll2a to vertebrates Vgll2 sequences. In situ hybridization showed that the two investigated Vgll2 genes had a similar expression pattern: they were first detected in adaxial cells (11 hpf), than expanded laterally in somites and at the end of segmentation, both genes were expressed in additional structures including head muscles and fin buds. In addition, different expression patterns of the two genes were observed. Vgll2a was expressed in bronchial arches precursor streams, derived gill muscles and hypothalamic precursors. Vgll2b was expressed in notochord at $14 \mathrm{hpf}$ and regressed following notochord maturation at $18 \mathrm{hpf}$. Furthermore, the genetic regulation of vgll2 genes was analysed using smu mutants and data revealed that both genes are regulated via the Hedgehog signaling pathway.
\end{abstract}

Keywords Zebrafish; Daniorerio, Vgll2, Vgl2, Vestigial, Somite, Myogenesis, Skeletal Muscles, Jaws Muscles, Branchial Arches, Gill Muscles, Notochord, Hypothalamus

\section{Introduction}

Invertebrates and vertebrates vestigial $(v g)$ and vestigial-like $(\mathrm{vgl})$ genes are involved in embryonic patterning and cell fate determination.

In Drosophila, the nuclear protein Vestigial (Vg) is a cofactor of Scalloped (Sd) and plays a central role in the development and patterning of wings [1,2]. Several works reported that $\mathrm{Vg}$ is also involved in the specification of a subset of flight muscle. Indeed, Vgis expressed in myoblast that will contribute to indirect flight muscle and controls the expression of Cut in the direct flight muscle [3,4]. In vertebrates, the expression of Tef1, the homologue of Sd, is not restricted to a specific tissue however it controls the expression of several genes during development in skeletal muscle and placenta through binding to a M-CAT element in regulatory regions of these genes [5-8]. This has lead to the hypothesis that tissue-specificity of Tef1 control is due to interaction of Tef1 with tissue-specific cofactor(s). Four genes have been identified in mouse which contains a sequence motif highly related to the Sd/Tef1 Interaction Domain (SID) of Vg. These genes have been named vgll1, vgll2, vgll3 and vgll4. vgll1 and vgll3 are expressed in placenta [9] while vgll2, also known as VITO1, is expressed in developing somites and muscle, in branchial arches and in limb buds $[6,10,11]$. The fourth gene, vgll4 has been cloned that has a widespread expression but is the only vertebratesvgllgene expressed in heart muscle [12]. Functional studies have revealed that Vgll2 and Vgll4 interact with Tef-1 through their SID domain and with Myocyte enhancer factor 2 (Mef2) [10-12]. Vgll4 modulates Tef1 activity in vitro [12] and Vgll2 activates muscular differentiation and is translocated in the nucleus during this process in vitro $[10,13]$. Thus Vgll2 appears to be the skeletal muscle specific cofactor of Tef- 1 in vertebrates. In addition, a recent report has described the expression of Vgll2 in the skeletal myogenic lineages of the chicken embryo under the control of myogenic factors [14].

In order to characterise genes involved in the control of muscle development, we have cloned vgll2 in zebrafish. Here, we report the cloning and expression of two zebrafishvgll2 genes. Both genes share high homology with mammalian Vgll2 and are expressed in body, head and fin bud muscles during embryonic development. In addition to this expression in muscle, vgll2a is also expressed in 
hypothalamic precursors and $v g l l 2 b$ is transiently expressed in notochord. Finally, we show that both genes are regulated by Hh signalling.

\section{Material \& Methods}

\subsection{Zebrafish Breeding}

Fish were obtain from a local pet shop and were breed following classical condition with a $14 \mathrm{~h} / 10 \mathrm{~h}$ day/night period. Embryos were obtained from pair mating and developmental stages determined according to Kimmel [27].

\subsection{Molecular Biology}

PCR primers were deduced from sequence of zebrafish ESTs and genomic fragments sharing homology with mouse Vgll2 identified in databases Ensembl[28]and WashU-Zebrafish Genome Resources Project [29]. mRNA were extracted from embryos of mixed developmental stages in the segmentation period using and Trizol reagent (Life Technologies). RT-PCR were performed with $1 \mu \mathrm{g}$ of total RNA using ExpandRT (Roche) followed by PCR reaction (Qbiogene). PCR fragments were cloned in PGEM-T vector (Promega). Sequences were performed using BigDye v1.1 fluorescent sequencing kit from Applied Biosystems.

Alignments were performed with the ClustalX program [30] with default settings. Alignment was decorated with Boxshade software [31]. Vgll2a and vgll2b names have been approved by Zebrafish gene name nomenclature committee.

\subsection{In Situ Hybridisation}

Antisense DIG probes were synthesised with appropriate RNA polymerase (Roche). In situ hybridisations were performed as previously described [24] and signal revealed with NBT/BCIP (Roche).

Gene's expression in wild-type and smu mutants has been quantified using ImageJ. Image was converted to greyscale after background subtraction and then expression intensity quantified in somites using the Fire LUT (Schröter et al., 2012). Statistical significance of relative expression was evaluated using a Mann-Whitney non-parametric test (Prism 6.0, Graphpad) [32].

\section{Results and Discussion}

\subsection{Molecular Characterisation of Two Vgll2 Genes in Zebrafish}

We identified two putative vgll2-related sequences in Ensembl and ESTs WashU databases. We deduced primers from these sequences and used them to clone corresponding cDNAs by RT-PCR. Both cDNAs encode a protein containing a SID and sharing high homology with vertebrates Vgll2 (Fig. 1A). The two zebrafish Vgll2s share a rather weak identity (39\%). One deduced peptide appears to be much closer to vertebrate Vgll2 and was named Vgll2a. Vgll2a shares 64 to $72 \%$ identity with vertebrates Vgll2. The second gene was named $v g l l 2 b$, the deduced peptide (Vgll2b) shares 39 to $43 \%$ identity with vertebrate Vgll2. When comparison is restricted to the putative SID domain, conservation of zebrafish Vgll2 is much higher with vertebrate Vgll (92 to 100\%) and with Drosophila Vg, 67\% and $71 \%$ for Vgll2a and Vgll2b respectively (Fig. 1B). Vgll2a and Vgll2b SIDs share 96\% identity. Sequence homology with other Vgll is limited to the SID (not shown).

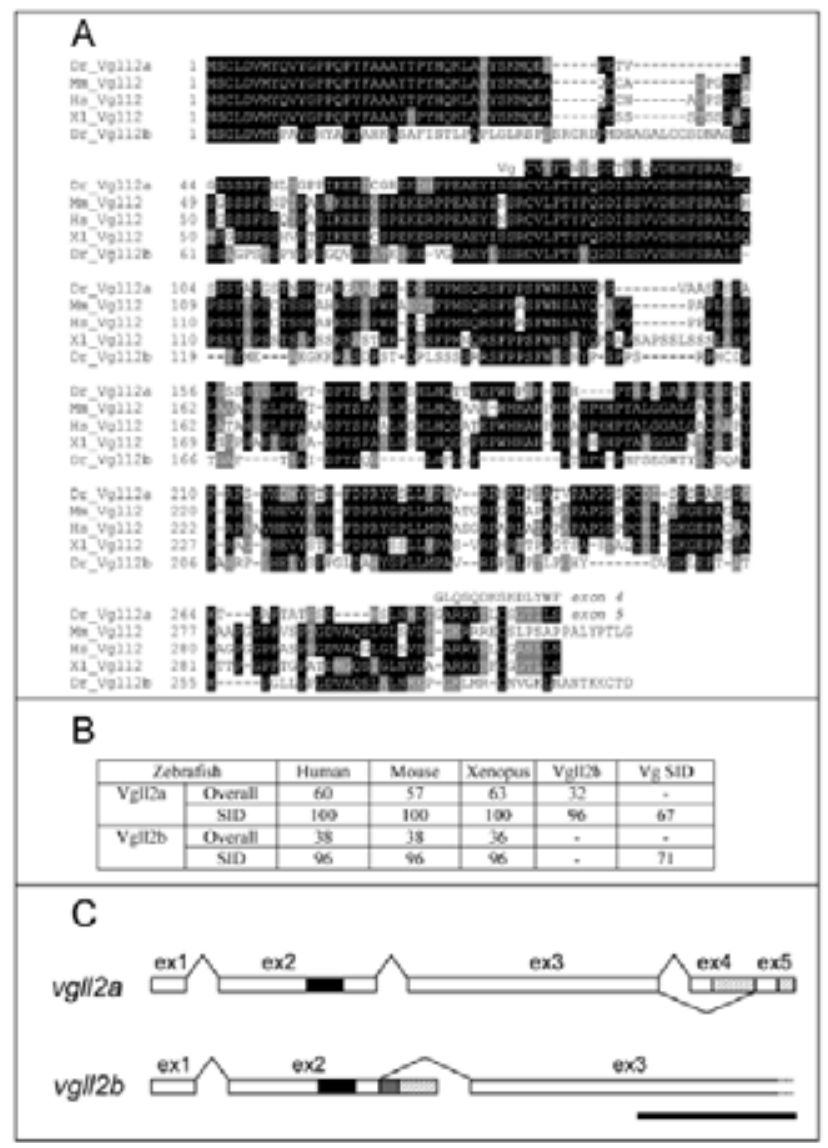

Figure 1. Characterisation of zebrafish Vgll2 and vgll2 genes organisation.

A) Alignment of zebrafish Vgll2 with their vertebrates counterparts and with drosophila Vg SID (Dr: Daniorerio; Mm: Musmusculus; Hs: Homo sapiens; Xl: Xenopuslaevis. Identical residues in all sequences appear black boxed. Grey shading marks similar residues. All vertebrate Vgll2 display almost strict conservation at the level of the SID and strong identity with Drosophila Vg SID. Vgll2a alternate peptide encoded by exon 4 is presented above peptide deduced from exon 5 which is closely related to human and XenopusC-terminus. B) Comparison of vertebrate Vgll2 sequences and identity at the level of SID. Percentage of identity was deduced from the alignment shown in A. Homology is even stronger in the functional SID domain including drosophila Vg SID domain. This comparison clearly assigns Vgll2a as a closer relative to vertebrates Vgll2 than Vgll2b. C) Genomic structure of zebrafishvgll 2 genes. SID is indicated in exon 2 of both genes as a black box. 3' untranslated regions are indicated as hatched boxes. vgll2agene contains 5 exons. Exons 4 and 5 are alternatively used. In the cDNA containing exon 4, exon 5 is included in the 3' untranslated region. Exon 5 encodes a C-terminus peptide highly homologous with xenopus and human Vgll2 C-terminus (see A). vgll2b contains at least 3 exons. Exon 3 incompletion is indicated as a dotted open box in 3'. The grey box after $v g l l 2 b$ exon 2 corresponds to the readthrough alternate transcript. The significance of this readthroughcDNA is not clear (see text). The black bar under gene schemes represent 100 base pair length. 
From RT-PCR experiments, we obtained two cDNAs for vgll2a differing only by their 3' extremities. Comparison with genomic sequence information from Ensembl database reveals that they are obtained after alternate splicing of exon 4 and 5 (Fig 1C). The peptide deduced from exon 5 shows high homology with the C-terminus of Xenopusand human Vgll2 (Fig. 1A). When exon 4 is used, exon 5 is included in the 3'untranslated region and both cDNAs use the same polyadenylation site. Vgll2b gene structure is simpler with only 3 exons, however, no stop codon has been found within the open reading frame. Comparison of cDNA and genomic sequences suggests that the oligo-dT oligonucleotide used for reverse transcription has primed in a A-rich region of the cDNA. Presented sequence is thus partial. The sequence of a $v g l l 2 b$ EST suggests that an alternate transcript with a readthrough of genomic sequence located after exon 2 may exist. We searched for such an alternate splicing by 3' RACE strategy and identified such a cDNA. In situ hybridizations performed with probes covering vgll $2 b$ exon 1 and 2 , vgll $2 b$ exon 3 and all three exons (see Fig. 1C) reveal the same expression pattern indicating that if this readthrough transcript has a physiological significance, it is not associated with a specific tissue expression (not shown). It is to note that even if gene organization of vgll2a and $v$ gll $2 b$ is similar, a first short exon and a second exon containing the SID, splicing sites are not conserved between these genes (Fig. 1C).

\subsection{Expression during Development}

We have analysed spatial and temporal expression pattern of zebrafishvgll $2 a$ and $v$ gll $2 b$ by whole mount in situ hybridisation during embryonic development up to $72 \mathrm{hpf}$ using labelled antisense riboprobes. Both vgll2 genes are first detectable at the 4-somite stage ( 11.5 hpf) in somites, in adaxial cells lining the notochord (Fig. 2A, B). This expression pattern extends as new somites are formed (Fig. 2C, D) and is similar to that of myogenin and of all three mef2 genes identified in zebrafish albeit vgll2 genes expression timing is closer to mef $2 D[15,16]$. Expression of vgll2 in cardiac precursors has never been detected. This observation is similar to the one made in developing mouse embryos and contrast with mef $2 A$ and mef $2 C$ expression which are detectable in this structure by 14 hpf[15]. As somites mature, expression extends laterally in somites from $15 \mathrm{hpf}$ (Fig. 2E -H). Expression in whole somite is still observed at $24 \mathrm{hfp}$ and decreases by $36 \mathrm{hpf}$. By $72 \mathrm{hpf}$ both vgll2 genes are expressed in fin buds mesenchyme (Fig. 2K, L).

In somites, vgll2 genes expression pattern is similar to the one of myogeninand mef2 myogenic factors $[17,18]$. This is in agreement with the involvement of Vgll2 in the control of muscle differentiation program suggested in mouse [10]. This expression is also similar to the recent study in xenopus embryos, where Vgll2 is expressed in the skeletal muscle lineage downstream of myogenic factors [19].

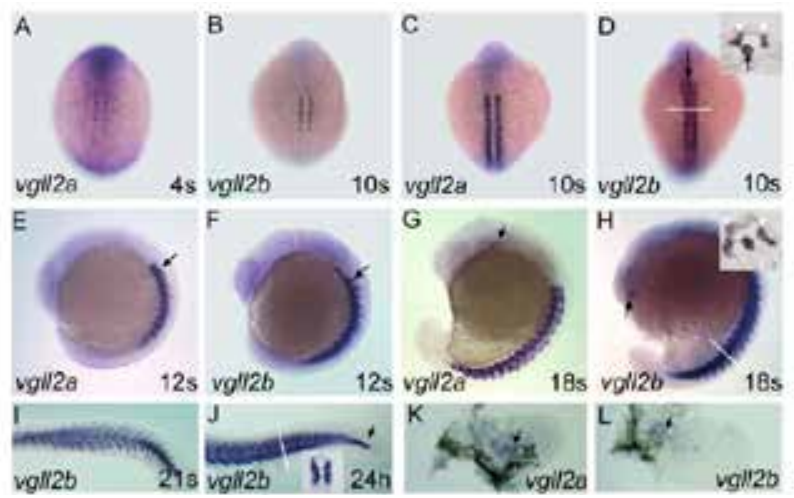

Figure 2. Early expression pattern of zebrafishvgll2 genes.

$v$ gll2a (A) and $v$ gll2 $b$ (B) are first detectable at the 4-somite stage in adaxial cells. (C, D) vgll2 expression extends to new somites as they are formed. At 10 -somite stage vgll $2 b$ is expressed in the anterior aspect of notochord (arrowhead in D; and insert: section at the level of the white line). (E, F) At the 12-somite stage, vgll2 genes expression enlarges in lateral somites from anterior to posterior somites. Vgll2b is now expressed in whole notochord (F). (G, H) At 18-somite stage, vgll2 expression is detected in whole somites (arrows). From this stage, vgll2a is also expressed in branchial arches precursor's streams (arrow in G) and vgll2b is expressed in an anterior bilateral cluster (arrow in $\mathrm{H}$ ). Insert in $2 \mathrm{H}$ : section performed at the level of the white line showing expression in somites (white arrowhead) and notochord. (I, J) Lateral view reveals at 21-somite stage that expression of $v g l l 2 b$ regresses in anterior notochord (I). At $24 \mathrm{hpf}$ expression in notochord remains only in the tip of the tail (arrow in J). Insert in J, section at the level of yolk extension (white line in $\mathrm{J}$ ) reveals expression in somites and the absence of expression in notochord. (K, L) Expression of vgll2a and vgll2b can be seen in mesenchyme of developing fin buds dissected out from $72 \mathrm{hpf}$ embryos. The shown phenotype is representative of $95 \%$ of the embryos, $n=$ 70

At the 10-somite stage (14 hpf) vgll2b begins to be expressed in anterior part of the notochord (Fig. 2D) and rapidly in the entire notochord (Fig. 2F,H). From 18hpf, expression regresses in the anterior aspect of notochord (Fig. 2I). This expression remains in the tip of the tail until $24 \mathrm{hpf}$ (Fig. 2J). This dynamic expression appears to be a novel function acquired by vgll $2 b$ since Vgll2 expression has never been detected in axial structure in mouse during development. We have never detected vgll2a expression in notochord.

From the 18-somite stage, vgll2 genes are also expressed in several developing head structures. Vgll2a is expressed in branchial arches precursor's streams from 18-somite stage (Fig. 2G) and in eye muscle at later stages (Fig. 3C-F). From 24 hpf, vgll2a is also expressed in hypothalamus precursors (Fig. 3A). By 36 hpf, vgll2a expression in hypothalamus is more precisely seen in pre-optic hypothalamus and in migrating neuro-hypophysis precursors (Fig. 3C). This pattern is similar to the one of xenopus Vgll2 where it occurs in the branchial arches and the stomodeal-hypophyseal anlage [19]. Expression in these territories persists until 72 hpf (Fig. 3D-F). This striking expression reminds the expression of another myogenic factor, myf5, in mouse hypothalamus [20]. From the 18-somite stage, vgll2b is expressed in bilateral clusters in the head (Fig. 2H, 3G). At $48 \mathrm{hpf}$, expression is detected in several head muscle precursors (Fig. 3H). At 72 hpf, these muscles can clearly be identified as jaw muscles and eye muscles (Fig. 3I). It is to note that in head, vgll2 genes are expressed in mutually 
exclusive subsets of muscle suggesting they acquired specialized functions. A recent report has detected zebrafishvgll $2 a$ expression in the pharyngeal endoderm and ectoderm surrounding the neural crest derived mesenchyme of the pharyngeal arches [21].

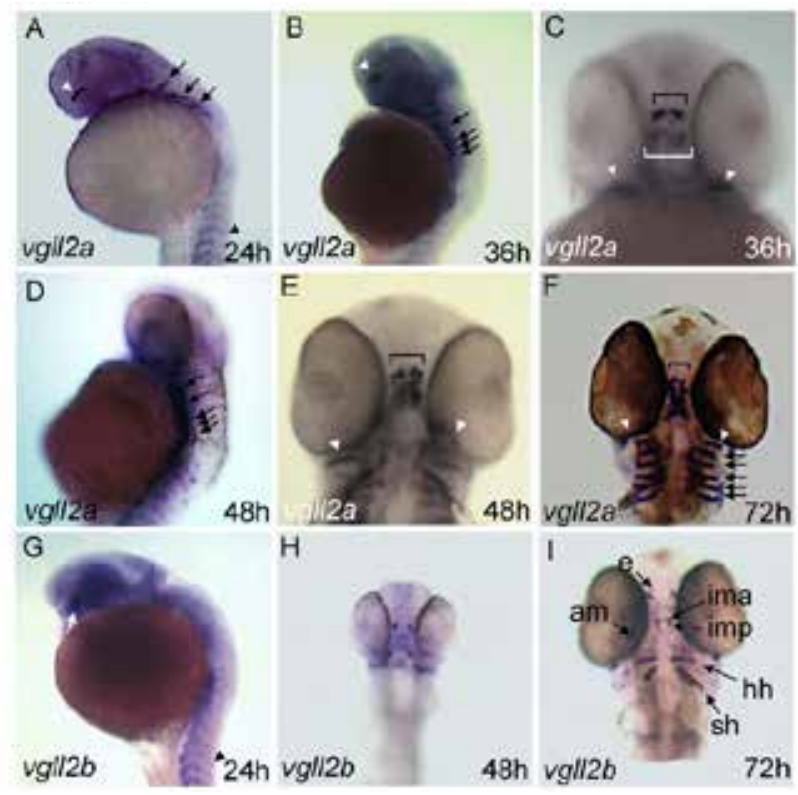

Figure 3. Expression pattern of zebrafishvgll2 genes in the head.

(A) Lateral view of $24 \mathrm{hpf}$ embryos showing vgll2a expression in the head. At this stage, $v g l l 2 a$ is expressed in migrating branchial arches streams (white arrows) and is still weakly expressed in somites (arrow). A new domain of expression appears in the head in hypothalamus territory (white arrowhead). (B) Expression is similar at $36 \mathrm{hpf}$. (C) Frontal view of a $36 \mathrm{hpf}$ embryo showing expression in eye muscle (white arrows) and hypothalamic precursors (brackets). The anterior cluster of cells (black bracket) constitute the pre-optic component of the hypothalamus, while the posterior one (white bracket) represents the precursors of developing neuro-epiphysis. (D-F) At 48 and $72 \mathrm{hpf}, v g l l 2 a$ is expressed in developing gill muscles (white arrows) and eye muscles (white arrowheads) and hypothalamus (brackets). (G) At $24 \mathrm{hpf}, v g l l 2 b$ is expressed in head muscle precursors and is still visible in somites. (H) Frontal view of a $48 \mathrm{hpf}$ embryo reveals expression of vgll $2 b$ in jaw and eye muscle precursors. (I) In this frontal view of a 72 hpf embryo, $v g l l 2 b$ expression can be seen in several jaw and eye muscles. am, adductor mandibulae; e, eye; hh, hyohyoideus; ih, inter hyoideus; ima, inter

mandibularis anterior; imp, inter mandibularis posterior; io, inferior oblique; ir, inferior rectus; mr, medial rectus. The shown phenotype is representative of $96 \%$ of the embryos, $n=76$

\subsection{Genetic Control of vgll2 Expression}

To identify genetic pathways that could be involved in the control of vgll2 genes expression. We analyzed the expression of vgll2 genes in acerebellar(ace) and slow muscle omitted (smu) mutants. acemutation results in the production of an inactive Fgf8 [22] and an altered fast myogenesis[23,24]. Expression of vgll2 genes appears unchanged in ace mutants indicating they are not regulated by Fgf8 (not shown). Smoothened is part of the Hedgehog (Hh) receptor complex and is mutated in smumutant [25,26], this mutation produces a blockade of all Hhsignalling and, among other phenotypes, in a suppression of myoDexpression in adaxial cells and then of slow muscle development [25]. We analyzed the expression of vgll2 genes at the 15-somite stage, when these genes are both expressed in adaxial and somitic muscle progenitors. In both cases, expression in adaxial cells is suppressed in smuembryos (Fig 4). Strikingly, vgll2b is also suppressed in somites (Fig. 4D). The mechanism underlying this regulation of vgll2b in fast muscle precursors by Hhsignalling remains unknown. Expression of vgll2b is not affected in notochord of smu embryos (Fig. 4D). Relative expression of vgll2a and vgll2b has been assessed in somites of smu mutants. Expression of vgll2a in smu embryos and wild-type were similar (relative expression $91.7 \pm 26.3 \% ; \mathrm{p}=0.4 ; \mathrm{n}=10$ ). Expression of vgll $2 b$ in somites of smu mutants was significantly lower compared to expression in wild-type embryos (relative expression $27.9 \pm 17.1 \% ; \mathrm{p}<0.01 ; \mathrm{n}=10$ ).

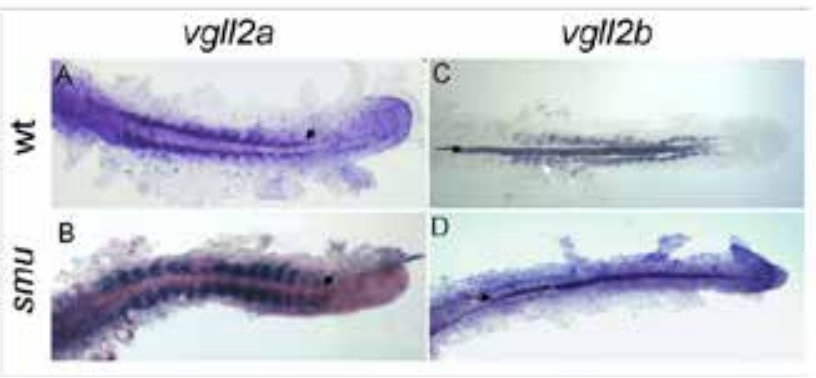

Figure 4. Expression of zebrafishvgll2 genes in Hh signalling devoid embryos.

Flat-mount embryos at 15-somite stage. (A, B) vgll2a expression is suppressed in adaxial cells of smuembryos. (C, D) vgll2b is also absent in adaxial cells of smuembryos, but more surprisingly, it is also absent in somites. Expression in notochord is not affected by absence of $\mathrm{Hh}$ signalling.

\section{Conclusion}

In summary, we have identified two zebrafishvgll2 orthologous. These genes are expressed in trunk and head muscles precursors during development. Expression in trunk muscle follows myogeninand mef2 genes expression timing. In the head, vgll2 genes are expressed in exclusive muscle, $v$ gll2a being expressed in gill and a subset of eye muscles while $v g l l 2 b$ is expressed in jaw and other eye muscles. In addition, vgll2 genes are expressed in non-muscle structures, $v g l l 2 a$ in ventral hypothalamus and vgll2b in notochord. Expression in such structures has never been described in mouse and reveals new functions acquired by these factors during evolution which deserve additional investigation. Finally, we have shown that in trunk muscle both vgll2 genes are regulated by $\mathrm{Hh}$ signalling, but again differentially. vgll $2 a$ is controlled by $\mathrm{Hh}$ in adaxial cells as are myoDor myogenin, while vgll $2 b$ requires $\mathrm{Hh}$ signal to be expressed in both adaxial cells and somites.

\section{Acknowledgements}

We thank Yannick Bidet for providing smuembryos. We thank Anne Bonnieu and Arnaud Chatonnet for fruitful 
discussions. This work was supported by grants from Département PHASE (INRA), Association Française de lute contre les Myopathies (AFM), EDST and National Council for Scientific Research, Lebanon (C.N.R.S.L). A.H. received a fellowship from PHASE (INRA) and Région Languedoc-Roussillon. C.G. received a fellowship from French Research Ministry (MRT).

\section{REFERENCES}

[1] de Celis JF. The function of vestigial in Drosophila wing development: how are tissue-specific responses to signalling pathways specified? BioEssays : news and reviews in molecular, cellular and developmental biology 1999;21:542-5.

[2] Kim J, Sebring A, Esch JJ, Kraus ME, Vorwerk K, Magee J, et al. Integration of positional signals and regulation of wing formation and identity by Drosophila vestigial gene. Nature 1996;382:133-8.

[3] Bernard F, Lalouette A, Gullaud M, Jeantet AY, Cossard R, Zider A, et al. Control of apterous by vestigial drives indirect flight muscle development in Drosophila. Developmental biology 2003;260:391-403.

[4] Sudarsan V, Anant S, Guptan P, VijayRaghavan K, Skaer H. Myoblast diversification and ectodermal signaling in Drosophila. Developmental cell 2001;1:829-39.

[5] Butler AJ, Ordahl CP. Poly(ADP-ribose) polymerase binds with transcription enhancer factor 1 to MCAT1 elements to regulate muscle-specific transcription. Molecular and cellular biology 1999;19:296-306.

[6] Jacquemin P, Oury C, Belayew A, Martial JA. A TEF-1 binding motif that interacts with a placental protein is important for the transcriptional activity of the hCS-B enhancer. DNA and cell biology 1994;13:1037-45.

[7] Jiang SW, Eberhardt NL. TEF-1 transrepression in BeWo cells is mediated through interactions with the TATA-binding protein, TBP. The Journal of biological chemistry 1996;271:9510-8.

[8] Stewart AF, Richard CW, 3rd, Suzow J, Stephan D, Weremowicz S, Morton CC, et al. Cloning of human RTEF-1, a transcriptional enhancer factor-1-related gene preferentially expressed in skeletal muscle: evidence for an ancient multigene family. Genomics 1996;37:68-76.

[9] Maeda T, Gupta MP, Stewart AF. TEF-1 and MEF2 transcription factors interact to regulate muscle-specific promoters. Biochemical and biophysical research communications 2002;294:791-7.

[10] Maeda T, Chapman DL, Stewart AF. Mammalian vestigial-like 2, a cofactor of TEF-1 and MEF2 transcription factors that promotes skeletal muscle differentiation. The Journal of biological chemistry 2002;277:48889-98.

[11] Mielcarek M, Gunther S, Kruger M, Braun T. VITO-1, a novel vestigial related protein is predominantly expressed in the skeletal muscle lineage. Gene expression patterns : GEP 2002;2:305-10.
[12] Chen HH, Mullett SJ, Stewart AF. Vgl-4, a novel member of the vestigial-like family of transcription cofactors, regulates alpha1-adrenergic activation of gene expression in cardiac myocytes. The Journal of biological chemistry 2004;279:30800-6.

[13] [13] Gunther S, Mielcarek M, Kruger M, Braun T. VITO-1 is an essential cofactor of TEF1-dependent muscle-specific gene regulation. Nucleic acids research 2004;32:791-802.

[14] Bonnet A, Lagarrigue S, Liaubet L, Robert-Granie C, Sancristobal M, Tosser-Klopp G. Pathway results from the chicken data set using GOTM, Pathway Studio and Ingenuity softwares. BMC proceedings 2009;3 Suppl 4:S11.

[15] Ticho BS, Stainier DY, Fishman MC, Breitbart RE. Three zebrafish MEF2 genes delineate somitic and cardiac muscle development in wild-type and mutant embryos. Mechanisms of development 1996;59:205-18.

[16] Weinberg ES, Allende ML, Kelly CS, Abdelhamid A, Murakami T, Andermann P, et al. Developmental regulation of zebrafish MyoD in wild-type, no tail and spadetail embryos. Development 1996;122:271-80.

[17] Mann CJ, Osborn DP, Hughes SM. Vestigial-like-2b (VITO-1b) and Tead-3a (Tef-5a) expression in zebrafish skeletal muscle, brain and notochord. Gene expression patterns : GEP 2007;7:827-36.

[18] Thisse B, Heyer V, Lux A, Alunni V, Degrave A, Seiliez I, et al. Spatial and temporal expression of the zebrafish genome by large-scale in situ hybridization screening. Methods in cell biology 2004;77:505-19.

[19] Faucheux C, Naye F, Treguer K, Fedou S, Thiebaud P, Theze $\mathrm{N}$. Vestigial like gene family expression in Xenopus: common and divergent features with other vertebrates. The International journal of developmental biology 2010;54:1375-82.

[20] Tajbakhsh S, Buckingham ME. Lineage restriction of the myogenic conversion factor myf-5 in the brain. Development 1995;121:4077-83.

[21] Johnson CW, Hernandez-Lagunas L, Feng W, Melvin VS, Williams T, Artinger KB. Vgll2a is required for neural crest cell survival during zebrafish craniofacial development. Developmental biology 2011;357:269-81.

[22] Reifers F, Bohli H, Walsh EC, Crossley PH, Stainier DY, Brand M. Fgf8 is mutated in zebrafish acerebellar (ace) mutants and is required for maintenance of midbrain-hindbrain boundary development and somitogenesis. Development 1998;125:2381-95.

[23] Groves JA, Hammond CL, Hughes SM. Fgf8 drives myogenic progression of a novel lateral fast muscle fibre population in zebrafish. Development 2005;132:4211-22.

[24] Hamade A, Deries M, Begemann G, Bally-Cuif L, Genet C, Sabatier F, et al. Retinoic acid activates myogenesis in vivo through Fgf8 signalling. Developmental biology 2006;289:127-40.

[25] Barresi MJ, Stickney HL, Devoto SH. The zebrafish slow-muscle-omitted gene product is required for Hedgehog signal transduction and the development of slow muscle identity. Development 2000;127:2189-99.

[26] Varga ZM, Amores A, Lewis KE, Yan YL, Postlethwait JH, 
Eisen JS, et al. Zebrafish smoothened functions in ventral neural tube specification and axon tract formation. Development 2001;128:3497-509.

[27] Kimmel CB, Ballard WW, Kimmel SR, Ullmann B, Schilling TF. Stages of embryonic development of the zebrafish. Developmental dynamics : an official publication of the American Association of Anatomists 1995;203:253-310.

[28] Stalker J, Gibbins B, Meidl P, Smith J, Spooner W, Hotz HR, et al. The Ensembl Web site: mechanics of a genome browser. Genome research 2004;14:951-5.
[29] http://zfish.wustl.edu/ OA.

[30] Chenna R, Sugawara H, Koike T, Lopez R, Gibson TJ, Higgins DG, et al. Multiple sequence alignment with the Clustal series of programs. Nucleic acids research 2003;31:3497-500.

[31] http://www.ch.embnet.org/software/BOX_form.html OA.

[32] Schröter C, Ares S, Morelli LG, Isakova A, Hens K, et al. Topology and Dynamics of the Zebrafish Segmentation Clock Core Circuit. PLoSBiol 2012;10(7):e1001364. 